DOI: $10.2478 /$ v10324-012-0005-4<smiles>CC1=CC=C1</smiles>

VERSITA
Analele Universităţii de Vest, Timişoara

Seria Matematică - Informatică L, 1, (2012), 69- 76

\title{
Interpolation of Locally Convex Triples
}

Dan Lupulescu and Ilie Stan

\begin{abstract}
In this paper some interpolation results are extended from locally convex couples to locally convex triples.
\end{abstract}

AMS Subject Classification (2000). 46M35

Keywords. Interpolation of locally convex spaces.

\section{Introduction}

The theory of interpolation usually deals with interpolation of two Banach spaces. In this case we mention, in particular the $K$ and $J$-methods (see e.g. [9],[1],[2],[3]). The paper by J.L. Lions and E. Magenes [8] incited to extend the theory of interpolation in the case of two locally convex spaces (see [4],[5],[6],[7]). In 1972 G. Sparr [10] extended the theory of interpolation to several (more than two) Banach spaces. The purpose of this paper is to extend some interpolation results from locally convex couples to locally convex triples.

\section{Preliminaries}

Let $\mathrm{C}_{2}$ be the category of locally convex triples. The objects of $\mathrm{C}_{2}$ are all triples $\vec{A}=\left(A_{0}, A_{1}, A_{2}\right)$ of Hausdorff locally convex spaces $A_{0}, A_{1}, A_{2}$ so that each $A_{i}(i=0,1,2)$ is linearly and continuosly embedded in a Hausdorff 
topological vector space $\mathcal{A}\left(A_{i} \hookrightarrow \mathcal{A}, i=0,1,2\right)$. Let $\vec{A}=\left(A_{0}, A_{1}, A_{2}\right)$ and $\vec{B}=\left(B_{0}, B_{1}, B_{2}\right)$ be two such triples then, by a morphism

$$
T: \vec{A} \rightarrow \vec{B}(T \in \mathcal{L}(\vec{A}, \vec{B}))
$$

we mean a linear mapping $T: A_{0}+A_{1}+A_{2} \rightarrow B_{0}+B_{1}+B_{2}$ so that $T_{/ A_{i}}$ (the restriction of $T$ to $\left.A_{i}\right)$ is continuous from $A_{i}$ into $B_{i}(i=0,1,2)$. Thus $\mathrm{C}_{0}$ is the category of Hausdorff locally convex spaces with morphisms defined by continuous linear mappings between such spaces. The set of all subspaces of a vector space forms, in a natural way, a lattice. Thus we can speak of the sum: $\Sigma(\vec{A}):=A_{0}+A_{1}+A_{2}$ and the intersection: $\Delta(\vec{A}):=A_{0} \cap A_{1} \cap A_{2}$. The topology of $\Sigma(\vec{A})$ is defined by the inductive limit of the $A_{k}$ (by $j_{k}$ : $\left.A_{k} \rightarrow \sum(\vec{A}), k=0,1,2\right)$. On $\Delta(\vec{A})$ we consider the projective topology, with respect to canonical imbeddings $i_{k}: \Delta(\vec{A}) \rightarrow A_{k}(k=0,1,2)$. Thus, $\Sigma(\vec{A})$ and $\Delta(\vec{A})$ are objects in $\mathcal{C}_{0}$. Now, we define two basic covariants functors: $F_{\Sigma}:=\Sigma(\operatorname{sum})$ and $F_{\Delta}:=\Delta$ (intersection) from $\mathcal{C}_{2}$ to $\mathcal{C}_{0}$ as follows:

$$
\begin{gathered}
\vec{A}=\left(A_{0}, A_{1}, A_{2}\right) \rightarrow F_{\Sigma}(\vec{A}):=A_{0}+A_{1}+A_{2} \\
T \in \mathcal{L}(\vec{A}, \vec{B}) \rightarrow F_{\Sigma}(T)=T: A_{0}+A_{1}+A_{2} \rightarrow B_{0}+B_{1}+B_{2}
\end{gathered}
$$

and

$$
\begin{gathered}
\vec{A}=\left(A_{0}, A_{1}, A_{2}\right) \rightarrow F_{\Delta}(\vec{A})=A_{0} \cap A_{1} \cap A_{2} \\
T \in \mathcal{L}(\vec{A}, \vec{B}) \rightarrow F_{\Delta}(T)=T: A_{0} \cap A_{1} \cap A_{2} \rightarrow B_{0} \cap B_{1} \cap B_{2}
\end{gathered}
$$

Indeed, if $T \in \mathcal{L}(\vec{A}, \vec{B})$ then $T$ is continuos from $A_{i}$ into $B_{0}+B_{1}+B_{2}$ $(i=0,1,2)$, and from the definition of the inductive limit topology, it is continuos from $A_{0}+A_{1}+A_{2}$ into $B_{0}+B_{1}+B_{2}$. Analogously, $T_{/ A_{0} \cap A_{1} \cap A_{2}}$ is continuos from $A_{0} \cap A_{1} \cap A_{2}$ into $B_{0} \cap B_{1} \cap B_{2}$.

Definition 2.1. By an interpolation functor on $\mathcal{C}_{2}$ we mean a functor

$$
F: \varrho_{2} \rightarrow \mathcal{C}_{0}
$$

so that:

(i) $\Delta(\vec{A}) \hookrightarrow F(\vec{A}) \hookrightarrow \Sigma(\vec{A})$ for any object in $\mathcal{C}_{2}$.

(ii) $F(T)=T_{F(\vec{A})}$ for any $T \in \mathcal{L}(\vec{A}, \vec{B})$ in $\mathcal{C}_{2}$. 
Definition 2.2. Let $\vec{A}=\left(A_{0}, A_{1}, A_{2}\right)$ be a given triple in $\mathrm{C}_{2}$. Then a space $A$ in $\mathcal{C}_{0}$ will be called an interpolation space with respect to $\vec{A}$ if there is an interpolation functor $F$ on $\mathcal{C}_{2}$ so that $F(\vec{A})=A$.

If in Definition 2.1 we consider only one-dimensional operators $T$ i.e.

$$
T a=f(a) b, b \in \Delta(\vec{A}), f \in(\Sigma(\vec{A}))^{*}
$$

then the space $A$ is called partly interpolation.

Now we will define a suitable "function norm". Let $\mathcal{M}$ be the set of all numerical (finite or infinite) functions $\varphi=\varphi\left(t_{1}, t_{2}\right)$ on $(0, \infty) \times(0, \infty)$ measurable with respect to the measure $\frac{d t_{1}}{t_{1}} \frac{d t_{2}}{t_{2}}$. We denote by $\mathcal{M}_{+}$the set of positive functions in $\mathcal{M}$. Generally, by a function norm on $\mathcal{M}_{+}$we mean a functional $\Phi$ defined for all $\varphi \in \mathcal{M}_{+}$with values in $[0, \infty]$ which has the following properties:

N1. $\Phi(\varphi)=0$ if and only if $\varphi=0$ a.e. ,

N2. $\Phi(\varphi)<\infty$ implies $\varphi<\infty$ a.e. ,

N3. $\Phi(c \varphi)=c \Phi(\varphi),(c>0)$,

N4.

$$
\varphi \leq \sum_{i=0}^{\infty} \varphi_{i} \quad \text { a.e. implies } \quad \Phi(\varphi) \leq \sum_{i=0}^{\infty} \Phi\left(\varphi_{i}\right)
$$

But our intention is to define interpolation functors $\mathcal{K}_{\Phi}$ which will be done by applying a suitable chosen function norm $\Phi$ on the Peetre $\mathcal{K}$-functional, so we need the following condition

N5. $\Phi\left(\min \left(1, t_{1}, t_{2}\right)\right)<\infty$

Example 2.1. Let $1 \leq p \leq \infty, \bar{\theta}=\left(\theta_{1}, \theta_{2}\right) \in(0,1) \times(0,1)$ with $\theta_{1}+\theta_{2}<1$. We define a function norm $\Phi$ (denoted in this case $\Phi_{\bar{\theta}, p}$ ) by

$$
\Phi_{\bar{\theta}, p}(\varphi)=\left(\iint_{\mathbb{R}_{+}^{2}}\left(t_{1}^{-\theta_{1}} t_{2}^{-\theta_{2}} \varphi\left(t_{1}, t_{2}\right)\right)^{p} \frac{d t_{1}}{t_{1}} \frac{d t_{2}}{t_{2}}\right)^{\frac{1}{p}} .
$$

If we put $R_{+}^{2}=D_{1} \cup D_{2} \cup D_{3} \cup D_{4}$ where: $D_{1}=(0,1) \times(0,1), D_{2}=(0,1) \times$ $[1, \infty], D_{3}=[1, \infty] \times[1, \infty]$ and $D_{4}=[1, \infty] \times(0,1)$, then

$$
\Phi_{\bar{\theta}, p}\left(\min \left(1, t_{1}, t_{2}\right)\right)=\left(\frac{1}{p^{2} \theta_{1} \theta_{2}\left(1-\theta_{1}-\theta_{2}\right)}\right)^{\frac{1}{p}}<\infty
$$




\section{$3 \quad$ Interpolation results}

Let $\vec{A}=\left(A_{0}, A_{1}, A_{2}\right)$ be an object in $\mathcal{C}_{2}$. The topology of $A_{0}$ is generated by the family of semi-norms $\left(p_{i}\right)_{i \in I}$, that of $A_{1}$ by $\left(q_{j}\right)_{j \in J}$ and that of $A_{2}$ by $\left(\rho_{k}\right)_{k \in K}$. We suppose that the families $\left(p_{i}\right)_{i \in I},\left(q_{j}\right)_{j \in J}$ and $\left(\rho_{k}\right)_{k \in K}$ are filled completed. For $\alpha \in A_{0}+A_{1}+A_{2}$, and $t_{1}, t_{2} \in(0, \infty)$ we consider the Peetre $\mathcal{K}$-functional, $\mathcal{K}_{i j k}$ asociated with the triple $\vec{A}$, i.e.

$$
\begin{gathered}
\mathcal{K}_{i j k}\left(t_{1}, t_{2}, a\right)=\inf \left\{p_{i}\left(a_{0}\right)+t_{1} q_{j}\left(a_{1}\right)+t_{2} \rho_{k}\left(a_{2}\right): a=a_{0}+a_{1}+a_{2}, a_{i} \in A_{i},\right. \\
i=0,1,2\}
\end{gathered}
$$

we have the folowing inequalities

$$
\begin{gathered}
\min \left(1, t_{1}, t_{2}\right) \mathcal{K}_{i j k}(1,1, a) \leq \mathcal{K}_{i j k}\left(t_{1}, t_{2}, a\right), \forall a \in A_{0}+A_{1}+A_{2} \\
\mathcal{K}_{i j k}\left(t_{1}, t_{2}, a\right) \leq \min \left(1, t_{1}, t_{2}\right) \max \left(p_{i}(a), t_{1} q_{j}(a), t_{2} \rho_{k}(a)\right), \forall a \in A_{0} \cap A_{1} \cap A_{2}
\end{gathered}
$$

Let $\Phi$ be a function norm. We put

$$
r_{i j k}(a)=\Phi\left(\mathcal{K}_{i j k}\left(t_{1}, t_{2}, a\right)\right)
$$

Theorem 3.1. Let $\vec{A}=\left(A_{0}, A_{1}, A_{2}\right)$ be an object in $\mathcal{C}_{2}$ and $\Phi$ a function norm. Then

$$
\mathcal{K}_{\Phi}: \mathcal{C}_{2} \rightarrow \mathcal{C}_{0}
$$

defined by $\mathcal{K}_{\Phi}(\vec{A})=\left\{a \in A_{0}+A_{1}+A_{2}: r_{i j k}(a)<\infty, \forall i \in I, j \in J, k \in K\right\}$ is an interpolation functor on the category $\mathcal{C}_{2}$.

Proof. At first we show that $\mathcal{K}_{\Phi}(\vec{A})$ is an Hausdorff locally convex spaces so that

$$
A_{0} \cap A_{1} \cap A_{2} \hookrightarrow \mathcal{K}_{\Phi}(\vec{A}) \hookrightarrow A_{0}+A_{1}+A_{2}
$$

Indeed the family $\left(r_{i j k}\right)_{(i, j, k) \in I \times J \times K}$ defines a family of semi-norms on the space $\mathcal{K}_{\Phi}(\vec{A})$ and the topology corespondent is finer than that of $A_{0}+A_{1}+A_{2}$ and les fine that of $A_{0} \cap A_{1} \cap A_{2}$. Let Now $\vec{B}=\left(B_{0}, B_{1}, B_{2}\right)$ be an object in $\mathrm{C}_{2}$. The topologies of $B_{0}, B_{1}$ and $B_{2}$ are generated by the filtrant families of semi-norms $\left(p_{l}^{\prime}\right)_{l \in L},\left(q_{m}^{\prime}\right)_{m \in M}$ and respective $\left(\rho_{s}^{\prime}\right)_{s \in S}$. Let $a \in \mathcal{K}_{\Phi}(\vec{A})$, $T \in \mathcal{L}(\vec{A}, \vec{B})$ and $(l, m, s) \in L \times M \times S$. We put

$$
r_{l m s}^{\prime}(T a)=\Phi\left(\mathcal{K}_{l m s}\left(t_{1}, t_{2}, T a\right)\right)
$$


But

$$
\begin{aligned}
\mathcal{K}_{l m s}\left(t_{1}, t_{2}, T a\right)= & \inf \left\{p_{l}^{\prime}\left(b_{0}\right)+t_{1} q_{m}^{\prime}\left(b_{1}\right)+t_{2} \rho_{s}^{\prime}\left(b_{2}\right), T a=b_{0}+b_{1}+b_{2}, b_{i} \in B_{i},\right. \\
& i=0,1,2\} \\
\leq & \inf \left\{p_{l}^{\prime}\left(T a_{o}\right)+t_{1} q_{m}^{\prime}\left(T a_{1}\right)+t_{2} \rho_{s}^{\prime}\left(T a_{2}\right), a=a_{0}+a_{1}+a_{2}, a_{i} \in A_{i},\right. \\
& i=0,1,2\}
\end{aligned}
$$

Since $T$ is continuos from $A_{n}$ into $B_{n}, n=0,1,2$ it follows that $\exists i(l) \in$ $I, j(m) \in J, k(s) \in K$ and $M>0$ so that

$$
\begin{aligned}
p_{l}^{\prime}\left(T a_{0}\right) & \leq M p_{i(l)}\left(a_{0}\right) \\
q_{m}^{\prime}\left(T a_{1}\right) & \leq M q_{j(m)}\left(a_{1}\right) \\
\rho_{s}^{\prime}\left(T a_{2}\right) & \leq M \rho_{k(s)}\left(a_{2}\right)
\end{aligned}
$$

Then

$$
\mathcal{K}_{l m s}\left(t_{1}, t_{2}, T a\right) \leq M \mathcal{K}_{i(l) j(m) k(s)}\left(t_{1}, t_{2}, a\right)
$$

and

$$
r_{l m s}^{\prime}(T a) \leq M r_{i(l) j(m) k(s)}(a)
$$

This means that $T$ is continuos from $\mathcal{K}_{\Phi}(\vec{A})$ into $\mathcal{K}_{\Phi}(\vec{B})$ and the proof is complete.

Theorem 3.2. Let $A$ be an partly interpolation space with respect to $\vec{A}=$ $\left(A_{0}, A_{1}, A_{2}\right)$ and $i, j, k \in\{0,1,2\}$ with $i \neq j \neq k$.

a) If $A \not \subset{\overline{A_{i}+A_{j}}}^{\Sigma(\vec{A})}$ then $A \supset A_{k}$.

b) If $A \not \subset{\overline{A_{i}}}^{\Sigma(\vec{A})}$ then $A \supset A_{j} \cap A_{k}$.

Proof. It is enough to prove a) and b) in the case $i=0, j=1, k=1$.

a) If $A \not \subset{\overline{A_{0}+A_{1}}}^{\Sigma(\vec{A})}$, there exists $a$ and $\varphi$, so that:

$$
0 \neq a \in A, a \notin{\overline{A_{0}+A_{1}}}^{\Sigma(\vec{A})}, \varphi \in(\Sigma(\vec{A}))^{*}, \varphi / \overline{A_{0}+A_{1}}=0 \text { and } \varphi(a)=1 .
$$

Let $b$ be an arbitrary fixed element of $A_{2}$. The linear mapping $T(x)=\varphi(x) b$ belongs to $\mathcal{L}(\vec{A}, \vec{A})$ and $b=T a \in A$, which finishes the proof of a).

b) If $A \subset{\overline{A_{0}}}^{\Sigma(\vec{A})}$ there exists $a$ and $\varphi$, so that:

$$
0 \neq a \in A, a \in{\overline{A_{0}}}^{\Sigma(\vec{A})}, \varphi \in\left(\Sigma((\vec{A}))^{*}, \varphi / \overline{A_{0}}=0 \text { and } \varphi(a)=1 .\right.
$$


Let $b$ be an arbitrary fixed element of $A_{1} \cap A_{2}$. The linear mapping $T x=$ $\varphi(x) b$ belongs to $\mathcal{L}(\vec{A}, \vec{A})$ and $b=T(a) \in A$.

Theorem 3.3. Let $\vec{A}=\left(A_{0}, A_{1}, A_{2}\right)$ be a locally convex triple. Let $C$ be a subspace of $A_{0}+A_{1}+A_{2}$. Then, for every interpolation functor $F$, we have

$$
F\left(A_{0} \cap C, A_{1} \cap C, A_{2} \cap C\right) \hookrightarrow F(\vec{A}) \cap C
$$

The spaces $A_{0} \cap C, A_{1} \cap C, A_{2} \cap C$ and $F(\vec{A}) \cap C$ are endowed with the topologies of $A_{0}, A_{1}, A_{2}$ and $F(\vec{A})$ respectively.

Proof. $A_{0} \cap C \hookrightarrow C, A_{1} \cap C \hookrightarrow C$ and $A_{2} \cap C \hookrightarrow C$ show that $\left(A_{0} \cap C, A_{1} \cap\right.$ $\left.C, A_{2} \cap C\right)$ is a locally convex triple. If $I$ denotes the embeding operator from $C$ into $A_{0}+A_{1}+A_{2}$, then $I \in \mathcal{L}\left(\left(A_{0} \cap C, A_{1} \cap C, A_{2} \cap C\right),\left(A_{0}, A_{1}, A_{2}\right)\right)$. Now, if $J$ denotes the embedding operator from $A_{0} \cap C+A_{1} \cap C+A_{2} \cap C$ into $C$, then $J \in \mathcal{L}\left(\left(A_{0} \cap C, A_{1} \cap C, A_{2} \cap C\right),(C, C, C)\right)$. Then by the interpolation property, we obtain (1).

Now we give a sufficient condition for obtaining the reverse inlusion in (1).

Theorem 3.4. Let $\vec{A}=\left(A_{0}, A_{1}, A_{2}\right)$ be a locally convex triple. Let $C$ be a subspace of $A_{0}+A_{1}+A_{2}$. If there is an operator $R \in \mathcal{L}(\vec{A}, \vec{A})$ so that:

i) $R\left(A_{0}+A_{1}+A_{2}\right)=C$

ii) the restriction of $R$ to $C$ is the identity

then, for every interpolation functor $F$, we have

$$
F\left(A_{0} \cap C, A_{1} \cap C, A_{2} \cap C\right)=F(\vec{A}) \cap C
$$

Proof. We have

$$
C=A_{0} \cap C+A_{1} \cap C+A_{2} \cap C
$$

So, $R \in \mathcal{L}\left(\left(A_{0}, A_{1}, A_{2}\right),\left(A_{0} \cap C, A_{1} \cap C, A_{2} \cap C\right)\right)$. Then, by the interpolation property, the restriction of $R$ to $F(\vec{A})$ is continous from $F(\vec{A})$ into $F\left(A_{0} \cap\right.$ $\left.C, A_{1} \cap C, A_{2} \cap C\right)$. Since the restriction of $R$ to $C$ is the identity we obtain

$$
F(\vec{A}) \cap C \hookrightarrow F\left(A_{0} \cap C, A_{1} \cap C, A_{2} \cap C\right)
$$

Corollary 3.4.1. Let $\mathcal{C}$ be a complemented subspace of $A_{0}+A_{1}+A_{2}$, whose projection $P \in \mathcal{L}(\vec{A}, \vec{A})$. Then (2) holds. 
Theorem 3.5. Let $\vec{A}=\left(A_{0}, A_{1}, A_{2}\right), \vec{B}=\left(B_{0}, B_{1}, B_{2}\right)$ be a locally convex triples and $T \in \mathcal{L}(\vec{A}, \vec{B})$. If there is an operator $S \in \mathcal{L}(\vec{B}, \vec{A})$ so that $T S=$ $I_{d} \vec{B}$ (the identity map) then, for every interpolation functor $F$, we have

$$
F\left(A_{0 T}, A_{1 T}, A_{2 T}\right)=F\left(A_{0}, A_{1}, A_{2}\right)_{T}
$$

where $A_{T}$ denotes $\left\{a \in A \hookrightarrow A_{0}+A_{1}+A_{2}: T a=0\right\}$ endowed with the A-topology.

Proof. We put

$$
\begin{gathered}
C=\left\{a \in A_{0}+A_{1}+A_{2}: T a=0\right\} \\
R=I_{d} \vec{A}-S T
\end{gathered}
$$

and then apply theorem 3.4.

\section{Application}

Let $A$ be a Banach space, and let $G(t)(t>0)$ be an strongly continuous semi-group of operators on $A$. $\mathbb{T}$ denotes the torrus $\mathbb{R} / 2 \pi \mathbb{Z}$ and $\mathrm{C}^{\infty}(\mathbb{T}, A)$ the space of all infinitely differentiable functions from $\mathbb{T}$ into $A$. Let $\left(M_{k}\right)_{k \in \mathbf{N}}$ be a positive sequence of real numbers and:

$$
A_{M_{k}}=A_{M_{k}}(\mathbb{T}, A)=\left\{f \in \mathfrak{C}^{\infty}(\mathbb{T}, A) ; \exists L>0: \sup _{k \in \mathbb{N}}=\frac{\left\|f^{(k)}\right\|_{L}^{2}(\mathbb{T}, A)}{L^{k} M_{k}}<\infty\right\}
$$

For $1 \leq s_{0} \leq s_{1} \leq s_{2}<\infty$ and $M_{k}=s_{0 k}$ !, $N_{k}=s_{1 k}$ !, $W_{k}=s_{2 k}$ ! we denote by $\dot{A}_{M_{k}}=\dot{A}_{M_{k}}(\mathbb{T}, A)=\left\{f \in A_{M_{k}}: f(0)=0\right\}$ and by analogy $\dot{A}_{N_{k}}, \dot{A}_{W_{k}}$. We define the morphism

$$
\mathcal{R}:\left(A_{M_{k}}, A_{N_{k}}, A_{W_{k}}\right) \longrightarrow\left(\dot{A}_{M_{k}}, \dot{A}_{N_{k}}, \dot{A}_{W_{k}}\right)
$$

by $\mathcal{R} f=f-(G \circ \varphi) f(0)$, where $\varphi(t)=\sin t, t \in \mathbb{T}$. Then there is an morphisms $\mathcal{S}:\left(\dot{A}_{M_{k}}, \dot{A}_{N_{k}}, \dot{A}_{W_{k}}\right) \longrightarrow\left(A_{M_{k}}, A_{N_{k}}, A_{W_{k}}\right)$ defined by $\mathcal{S} f=f$, such that $\mathcal{R S}=I_{d}$. Consequently, by Theorema 3.5. we obtain, for every interpolation functor $F$,

$$
F\left(A_{M_{k} \mathcal{R}}, A_{N_{k} \mathcal{R}}, A_{W_{k} \mathcal{R}}\right)=F\left(A_{M_{k}}, A_{N_{k}}, A_{W_{k}}\right)_{\mathcal{R}}
$$

Remark 4.1. In the case of couples, this example is given in [6]. 


\section{References}

[1] J. Bergh and J. Löfström, Interpolation spaces, An introduction, Springer-Verlag, Berlin, Heidelberg, New-York, 1976.

[2] Yu. A. Brudnyi and N. Ya. Krugljak, North-hooland, Amsterdam, 1991.

[3] P. L. Butzer and H. Berens, Semi-groups of operators and aproximation, Basel:Birkhäuser, 1972.

[4] N. Deutsch, Interpolation dans les espaces vectoriels topologiques localment convex, Mémoires de la S.M.F., 13, (1968), 3-187.

[5] C. Goulaouic, Prolongements de foncteurs d'interpolation et applications, Annales de l'institut Fourier, 18, nr.1, (1968), 1-98.

[6] C. Goulaouic, Interpolation entre des espaces localment convex définis à l'aide de semi-groups; cas des espaces de Gevrey, Annales de l'institut Fourier, 19, nr.2, (1969), 269-278.

[7] C. Goulaouic and M. S. Baouendi, Comutation de l'intersection et des foncteurs d'interpolation, de l'intersection et des foncteurs d'interpolation, C. R. Acad. Sc. Paris, t.265, 11 septembre 1967.

[8] J. L. Lions and E. Magenes, Espaces de fonctions et distributions du type de Gevrey et problémes aux limites paraboliques, LXVIII, (1965), 341-418.

[9] J. Peetre, A theory of interpolation of normed spaces, Notes,1963(=Notas de matematica 39), Brasilia, 1968.

[10] G. Spaar, Interpolation of several Banach spaces, Ann. Mat. Pura Appl. 990, (1974), 247-316.

Dan Lupulescu

West University of Timişoara

Bd. V. Pârvan, Nr. 4

Timişoara 1900

E-mail: danlupulescuv@gmail.com

Ilie Stan

Department of Mathematics

West University of Timişoara

Bd. V. Pârvan, Nr. 4

Timişoara 1900

E-mail: stan@math.uvt.ro

Received: 10.11.2012

Accepted: 25.07.2012 